\title{
Probabilistic Road-Aware Geocast In VANETs
}

\author{
Zubair Amjad*, Wang-Cheol Song** \\ Departement of Computer Engineering, Jeju National University \\ Jeju, South Korea \\ Email: *xubairamjad@gmail.com,**philo@jejunu.ac.kr
}

\section{Article Info}

Article history:

Received Jan 19, 2015

Revised Apr 22, 2015

Accepted May 8, 2015

\section{Keyword:}

Geocast

Location Service

Probability

Road data

Tree

VANET

\begin{abstract}
Geocast is a communication technique to disseminate information in specific geographic regions instead of node addresses. Traffic congestion, accidents, local hazards and digital content sharing are potential use cases of information sharing in VANETs. Recently, several approaches for geocast routing have been proposed to achieve high delivery ratios. These approaches consider a center point and radius to define the destination region also called geocast region. They focus only on routing scheme to enhance the delivery ratio and delays. However, these approaches do not consider the target region selection problem in the geocast routing. In this paper, we propose a novel application-level mechanism for sharing road conditions, such as accidents, detours and congestion in VANETs through probabilistic road-aware geocast routing. We assign probabilities to the roads around each intersection in the neighborhood road network of the source vehicle. We then build a spanning tree of roads (from graph representation of the road network) with information source as the root node. Nodes below the root represent junctions and edges represent inter-connecting road segments. Messages propagate along the branches of the spanning tree. The spanning tree represents the geocast region. As the information propagates down the branches, probability of road as geocast region decreases. Information is propagated until a threshold probability is reached. Our method also ensures that messages are not delivered to irrelevant vehicles irrespective of their proximity to the source. We evaluate our application through extensive and realistic simulations in ns-3 simulator using IDM car following and MOBIL lane change models for realistic modeling of vehicle mobility.
\end{abstract}

Copyright () 2015 Institute of Advanced Engineering and Science. All rights reserved.

\section{Corresponding Author:}

Wang-Cheol Song,

Departement of Computer Engineering,

Jeju National University,

102 Jejudaehak-ro, Jeju-si, Jeju Special Self-Governing Province, Republic of Korea, 690-756

Email: philo@jejunu.ac.kr

\section{INTRODUCTION}

Recently, Vehicular Ad-Hoc Networks (VANETs) have received much interest from automotive industry and the research community. VANETs are actively being used as a medium for sharing information among vehicles. Additionally, VANETs have also emerged as practical application model for research and development in the field of Mobile Ad-hoc Networks (MANETs). Majority of the work done for MANETs can easily be carried over to VANETs due to the similar implementation scenarios of the two. Some VANETs applications address issues of road safety and improving driving experience because of accidents, hazardous conditions and congestion related issues while others are built for entertainment purposes, e.g. multimedia content sharing including game content for entertainment. [1-4] show works for delivering safety related information or traffic congestion information to drivers to let them drive more safely on the roads, and 
[5-8] provide an entertainment by enabling interactive games, content sharing and dissemination of advertisements to interested clients.

For sharing road information with other vehicles, geocast [9] is considered beneficial as it enables the vehicles to send information to specific geographic areas. Simple flooding is useful as it sends geocast packet to every node irrespective of target region and receiver vehicles check whether they are in the destination area or not. In directed flooding [10], a geocast region (GR) is defined with a center point and a radius as the target region. Packets are flooded toward GR through forwarding zone which has forwarding nodes in it.

In [11-14] authors have proposed geocast routing protocols to disseminate information in VANETs. However, these protocols select the GR as a circular or rectangular region with a predefined center point. Target region selection affects the information availability among all candidate nodes as the distance between intersections is very short in urban areas and it is too large in the highways. Using a circular or rectangular geocast region with a fixed center point and radius can affect the delivery ratio in highway VANETs due to the larger road segment lengths. However, it can be very useful in the urban VANETs. It is important to disseminate the relevant road information among all nodes which are directed towards hazardous roads. Selecting a circular region as GR increases the probability of missing out some vehicles who are directed towards the region which has accident or congestion.

In geocast routing, vehicles broadcast messages towards GR to propagate information. In many proposed geocast routing protocols [11-14], the position information is used from GPS and navigation system. The source vehicle sends the geocast packet towards a geographic region and vehicles inside that region save that packet upon receiving. However, we believe that road information can be used, such as number of lanes and road IDs in order to provide an efficient road-aware geocast scheme. Number of lanes on a road can be useful in order to find probability of cars on a road going to another road. Since this information can easily be available to all vehicles through digital maps, we propose a geocast scheme which makes use of it. As vehicles may usually have a navigation system these days, it is assumed that vehicles can figure out their road information as well as driving information based on their current location received by GPS. Direction of vehicles can be considered as a parameter for this mechanism because when some information is generated at a particular point on the road, outgoing vehicles usually have no interest in it but incoming vehicles can be interested in that information. Every incoming vehicle should receive the relevant information in order to avoid the hazardous scenarios.

In this road-aware approach, we propose a probability based efficient geocast technique for VANETs. In order to apply the road information we have presented roads as a spanning tree and defined how and whom to deliver the information in the tree. Geocast region consists of roads directed towards the information source point such as an accident or congestion on the road. In our work we have treated the information source point asa fixed geographical location because we expect that our proposed system can be possibly used for information sharing in safety related cases and/or disaster situations only, and we do not consider a situation where an information source maypersistently move as in the case of multimedia or game content sharing.

As real world VANET experimentation is prohibitively expensive, we use NS-3 [15] simulation as the first step to evaluate our solution. NS-3 provides realistic implementation of network protocol stack based on Linux kernel implementation of the protocol stack. Furthermore, wireless models in NS-3 are more realistic. It is easy to further improve the realism by incorporating application, protocol stack or network interface level emulation by leveraging its Direct Code Execution (DCE) capability. As NS-3 is not tailored for VANET mobility scenarios, we have integrated IDM car following and MOBIL lane change models that realistically mimic mobility of vehicles. We test the working and performance of Wi-Fi based information sharing mechanism over a road network typical of modern urban center.

The paper is organized as follows: Section 2 introduces the related work and Section 3 describes the mechanism proposed in this paper. In Section 4, we present the evaluation to show the performance of our system. We conclude the paper in Section 5.

\section{RELATED WORK}

VANETs are different from other kinds of ad hoc networks by some characteristics, such as hybrid network architectures, node movements and new application scenarios [16]. VANET routing protocols can be classified into five categories as follows [17]: cluster based routing, ad-hoc routing, broadcast routing, position based routing, and geocast routing. In various aspects, VANETs have similar characteristics to MANETs. Some of the existing protocols of MANETs are appropriate for VANETs but most of these achieve low performance results due to highly dynamic characteristics of VANETs. Broadcast routing is the most used protocol in VANETs [17] but it causes bandwidth problems. 
Multicast is a routing scheme used to send packets to random group of nodes, e.g. all nodes who have subscribed for a particular service [9]. Geocast can be defined by a multicast group having a geographical area as destination because geocast is a subclass of multicast. In VANETs, geocast sends data packets from a single source vehicle to all vehicles residing in the destination area called geocast region (GR) $[18,19]$. Target vehicles vary due to their mobility while the target area remains the same. Directed Flooding [10] is a proposed geocast protocol that allows packet forwarding in the region which is closer to the destination than the sender. Directed flooding not only defines geocast region as target region but also defines forwarding region which is used by the vehicles to forward the packets. Vehicles can only broadcast the messages in the forwarding zone.

UGAD [12] is an adaptive delay based geocast protocol designed for urban environments. Vehicles broadcast messages in two different forwarding modes depending on their location. Vehicles on intersections have higher priority of rebroadcasting as compared to other vehicles. These priorities are assigned due to buildings which block transmissions. The dual mode hybrid approach is very effective in urban scenarios but it is not road-aware like our proposed solution and lacks the probabilistic efficiency which we have provided. This scheme only focuses on urban scenarios and may not be effective in highway road networks. The target region is selected as a circular region which is not suitable for sharing information in both urban and highway scenarios.

GeoSPIN [11] is an approach for geocast routing based on spatial information in VANETs. It provides a geocast routing method based on daily movements of vehicles. Every vehicle keeps its past raw data of trajectories to perform clusterization technique and gets the probabilities of it going to different roads. These probabilities are then used to select the best relayer among the candidate vehicles. Relayer vehicles forward the message whenever they find a vehicle with higher probability of going towards geocast region. This approach shows very effective results; however, this method increases the computations and requires the daily trajectories which may not be available for all the vehicles in any particular city.

Dynamic Time-Stable Geocast Routing in Vehicular Ad Hoc Networks (DTSG) [20] has proposed a geocast routing scheme to inform vehicles on a particular highway about certain events. GTSG assumes vehicles move with the same velocity which is not the case in reality. DTSG takes advantage of helping vehicles (Vehicles moving in the opposite direction) to send the message to different vehicles. There are two phases in this approach, pre-stable period and stable period. In pre-stable phase, vehicles broadcast message until it reaches at the end region. Stable phase allows the protocol stabilization within the region. These proposed geocast schemes [11-14] only focus on routing and do not consider the necessary road attributes like direction and lanes on the road which are pivotal for reducing significant transmission overhead on basis of relevancy. As vehicles going in opposite direction have no need of this information, these schemes do not take road-aware approach for information sharing in VANET.

In this paper, we try to overcome the above mentioned problem of geocast region selection. In our scheme, roads are selected as geocast regions based on the probability of the cars on a road going towards the hazardous roads. We describe the evaluation of our application in Section 4 and show the results.

\section{PROBABILISTIC ROAD-AWARE GEOCAST IN VANETS}

Existing work about geocast in VANETs considers only a center point and a radius as geocast region. Considering different road scenarios such as urban, highway or mountainous roads, the fixed radius circle as geocast region is not good approach. In case of an accident on the road, every car going towards the accident road should get the information while for the other vehicles going away from the accident, the information is irrelevant. However, opposite direction vehicles can be used to forward the packets towards the geocast regions. A circular geocast region approach can be ineffective on the roads where intersections have large separation distances. With these considerations in mind, in this paper, we propose a novel roadaware geocast mechanism to share road information in VANETs.

We assume that every vehicle is equipped with a navigation system. As these days many vehicles are equipped with navigation system, we think this assumption is reasonable. For the sake of simplicity, we consider the scenario of an accident on a road,as shown in figure 1. Obviously, accident information is relevant only to the vehicles travelling in the direction of the accident point and should be delivered to those vehicles only. In our mechanism, a vehicle having the information, broadcasts it to the neighboring vehicles. Vehicles which receive the information can discard that information if they do not reside in the geocast region. We assign priorities to different scenarios e.g. if an accident occurs on the road, information about it gets the highest priority while in case of congestion on the road, the priority of this information is lower. In case the message buffer of a vehicle is full, information with high probability is given high priority. 


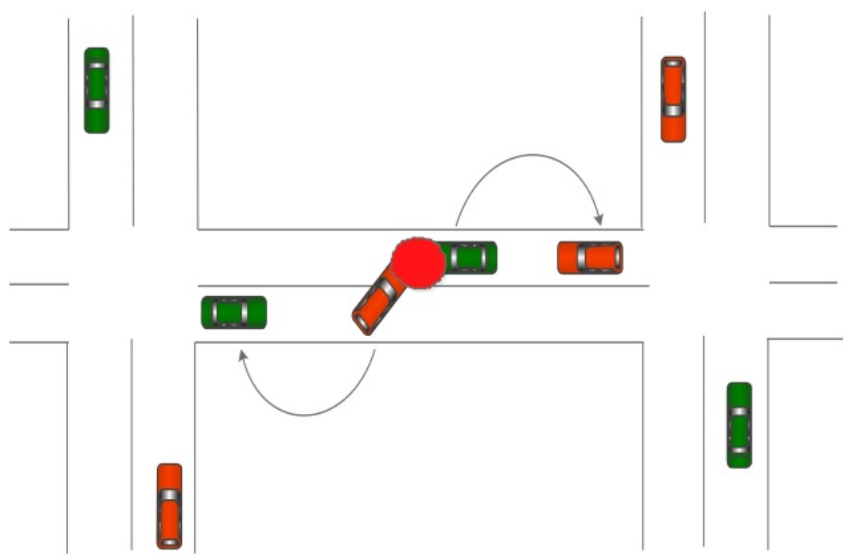

Figure 1. An Accident Occurred On A Road

When information is generated due to problems like traffic accidents, it is relevant to all vehicles travelling on that road. So, all vehicles on the road where an accident takes place need to receive the information without exception. If a road is very long without divergence likeroads in mountainous areas, the information should be delivered toall vehicles regardless of the distance. In urban areas, roads frequently meet a junction after a short distance; therefore the road information can be shared only in a small local area. As a special case, when a traffic accident occurs in a multiple-level complex interchange, the accident information should be delivered to all vehicles only on the roads where traffic can be affected by the accident. Our geocast mechanism works as follows. First, information is delivered to all vehicles on the same road as the accidentwith probability of 1 .

Then, we define how the information can be shared among vehicles on other roads connected via intersections. As the number of lanes of roads is decided based on the expected traffic load at the time of their construction, we can estimate the probability of which road a vehicle will select in an intersection by using the number of lanes.

Let us explain how to calculate the probability to select a road after a junction using figure 2 as an example. Four roads connected to a junction have different number of lanes respectively. If an accident occurs on road (1) and accident information is transmitted, all vehicles heading to the accident location on the same road should get the accident information with probability 1 , but for vehicles on other roads we need to know their probabilities of entering into road (1) across the junction. We propose to calculate the probability using number of lanes for the road. As mentioned before, when a road is being built, its expected traffic load is estimated and is translated to the number of lanes. So, we propose to use the number of lanes in the road, and equation 1 can be used to calculate the probability.Equation 1 expresses a simple ratio of the number of lanes for a road that a car selects over all possible roads into which a car will enter from the currently-riding road.

$$
\begin{gathered}
p_{i}=\frac{\text { number of lanes on the accident road }}{\text { total number of lanes of roads except road } i} \\
\text { where the car is }
\end{gathered}
$$

In figure 2, probability for a car to enter from road (2) into road (1) can be estimated by dividing the number of lanes of road (1) by the number of total lanes of roads (1), (3), (4) and (5), not in road (2).

$$
p_{2}=\frac{\text { number of lanes on the accident road (1) }}{\text { total number of lanes on roads }\{(1),(3),(4)\}}=\frac{3}{3+2+3}=\frac{3}{8}
$$




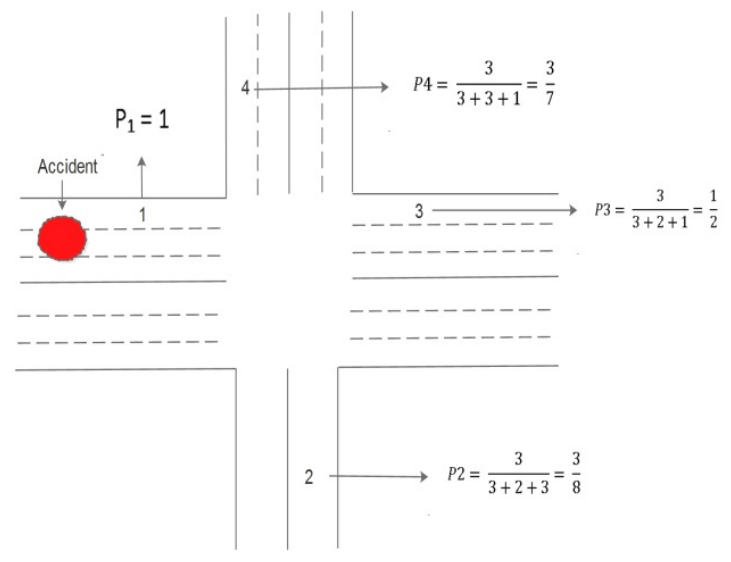

Figure2. Calculating Pi for Roads

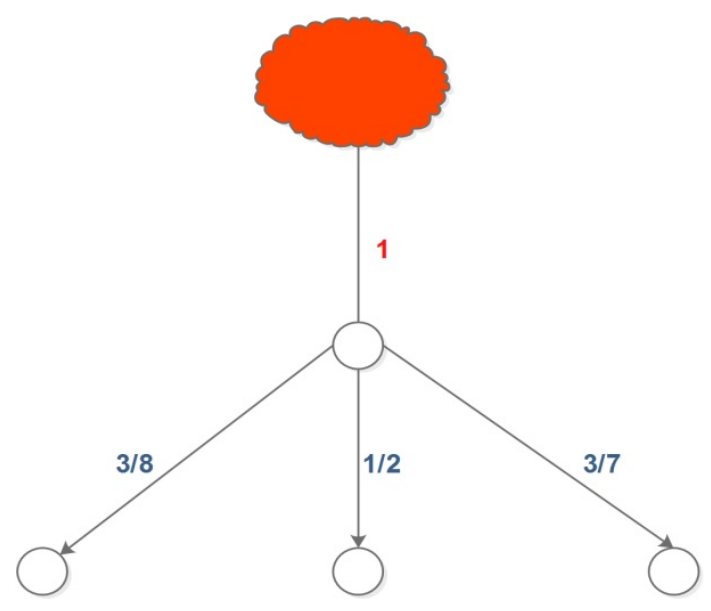

Figure 3.A Spanning Tree Representing Roads and Junctions

Then, we can say that the vehicles on road (1) can receive the information with probabilityp ${ }_{1}=1$, and vehicles on road (2) can receive it with probability $\mathrm{p}_{2}=3 / 8$.

In the same way, $\mathrm{p}_{3}=1 / 2$ and $\mathrm{p}_{4}=3 / 7$ can be calculated. Then, we can know that when an accident occurs on road (1), vehicles on roads (2), (3) and (4) are expected to go to road (1) with probabilities 3/8, 1/2 and 3/7 respectively. Therefore, with those probabilities, we can represent the roads using the spanning tree in figure 3. Also, we can extend the tree by calculating probabilities in the same way.

Now we need to consider the range to share the information by extending the spanning tree to more intersections. As mentioned, we need to specify it in term of related road probabilities $p_{\mathrm{i}}$. When we calculate it, we can calculate how much valid information is present on a road. When a road is far from the road where the information is generated (we can call it a source road) by crossing many junctions, validity of the information also essentially decreases. It can be expressed as a probability from a source road to a road and it is computed by multiplying all $p_{\mathrm{i}}$ from a source road to a current road using equation 2 .

$$
p_{\text {road_k }}=\prod_{i=1}^{k} p_{i}
$$

We can say that vehicles on a road $k$ have the probability $p_{\text {road_k }}=p_{1} \mathrm{x} \ldots \mathrm{x} p_{\mathrm{k}}$ which is product of probabilities $p_{\mathrm{i}}$ of roads along the path from the root to node $k$ in the spanning tree. The depth of the tree can be unlimited, but we limititusing a threshold probability. In this paper, we consider the roads as geocast region having $p_{\text {road_k }}>0.05$ for the sake of simplicity, but it may be decided differently based on its importance and emergency. As we can see, $p_{\text {road_k }}$ itself is relevant to the generated source information. In our mechanism, we use it to control how much information can be shared to neighbor vehicles. A vehicle having 
road informationshares it by periodically broadcasting it. When a vehicle $A$ meets another vehicle $B, A$ will geocast item $I$ to $B$ with probability $p_{\mathrm{r}}(k)$ :

$$
p_{\mathrm{r}}(k)=p_{\text {road_k }}
$$

The vehicles that have received the information decides whether it will be stored or dropped depending upon its status such as available memory in the receive buffer. We define the deletion probability $p_{\mathrm{d}}(k)$ in a similar way:

$$
p_{\mathrm{d}}(k)=1-p_{\text {road_k }}
$$

Where $\mathrm{k}$ is the road number, the replicated information can be assigned a priority level using probability and handled accordingly.

In this way, we have created a new method to specify a geocast region with the road-aware approachin a VANET by taking into account road information rather than distance from the source. As an example, we consider the case in figure 4. As the accident occurs in the center of a road, an alert should be deliveredto all the vehicles coming towards the accident point from both sides. We can calculate probability $p_{i}$ of the roads with respect to the accident point. Figure 5 showsprobability pi for each road i in figure 4 . Figure 5 also shows assumed probabilities assigned to extended roads in order to demonstrate the computation of $p_{\text {road_k }}$. The final probability tree is shown in Figure 6 . The depth of the tree is limited by the threshold 0.05 i.e., proad_k>0.05. The depth can be increased or decreased by choosing a different value for the threshold depending on the application scenario.

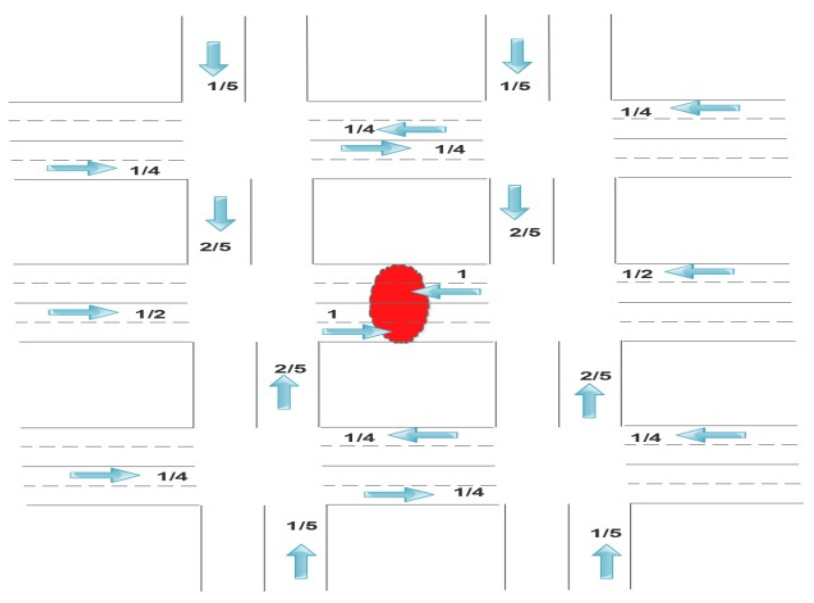

Figure 4. An Example When an Accident Occurs on A Road

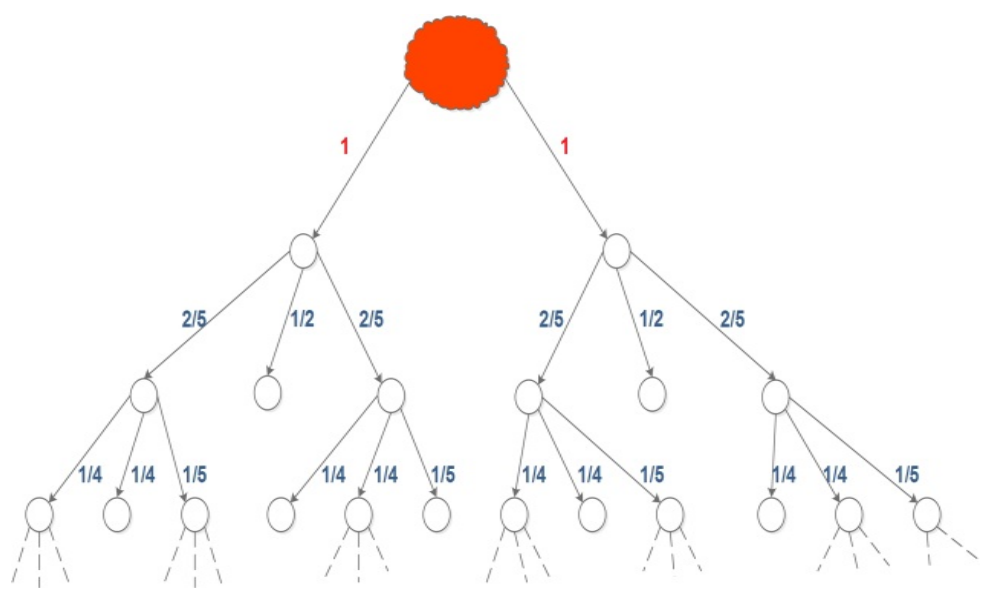

Figure 5. pi Calculated for Roads in Figure 4 
Figure 7 shows the selected geocast region after an accident as shown in figure 4. Messages are propagated along the spanning tree which is limited by the threshold. The selected geocast region is highlighted as well as the accident point. Our road-aware geocast approach is different from other geocast methods in term of selection of the GR. As it can be clearly seen from the figure 7, that only relevant roads are being selected for the message geocasting. Roads going away from the accident are excluded from the geocast region.

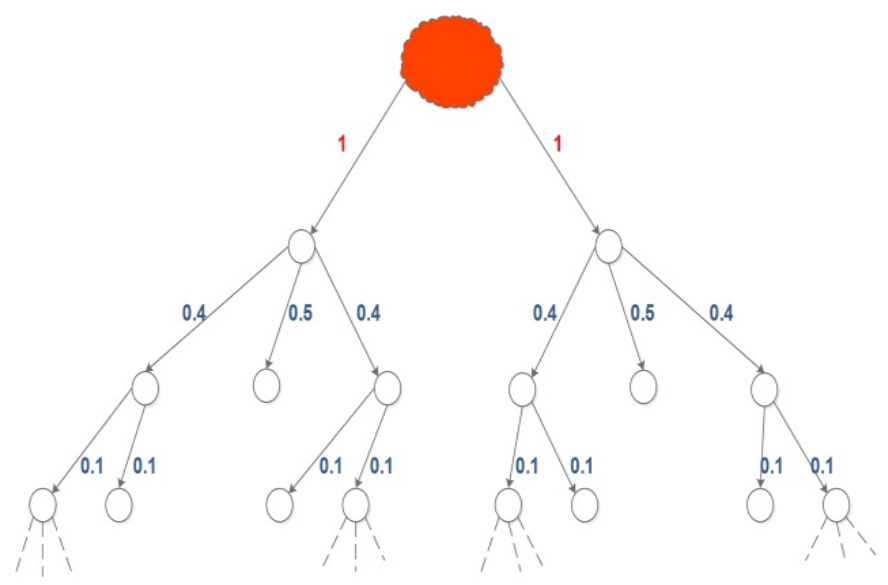

Figure 6. proad_k in a Spanning tree for proad_k>0.05

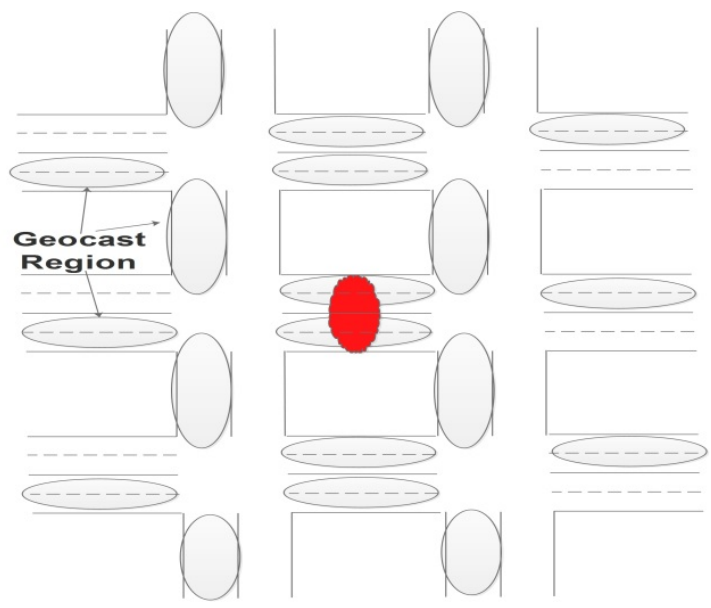

Figure 7. Selected Geocast Region after Spanning Tree Calculation

\section{EXPERIMENTATION}

In out simulations, we have assumed the situation of a traffic accident where accident vehicle originates the messages. Other vehicles accept these messages according to their road probability and forward it to their neighbors. Vehicles with probability less than the threshold value do not accept the messages as they do not reside in the geocast region. If a vehicle cannot find a neighbor to forward the message, it discards the message after its life time defined by the source vehicle.

The source vehicle generates a warning message with a lifetime (TTL). The message is stamped with its time of creation and is identified by a unique ID. Vehicles continuously send neighbor discovery beacons or hello messages to discover neighbors. Whenever a vehicle discovers a neighbor, it forwards the available message to that vehicle depending on the road probability of the neighbor vehicle. Road information of neighbor vehicle is available in hello messages. If neighbor vehicle has probability less than the threshold, the neighbor is not inside the geocast region. The messages are not forwarded to the vehicles outside GR. The sending vehicle prioritizes the order in which messages are shared according to a replication policy. The replication policy determines in which order messages are replicated when two vehicles come within the radio range of each other. Buffer on each vehicle is periodically evaluated to determine the 
messages to delete. This is to prevent buffers from filling up. Deletion function depends on the road probability. High road (replication probability) means less deletion probability and is computed as 1 - $\mathrm{P}_{\text {road_k }}$ where road is number of the road and $\mathrm{P}\left(\mathrm{P}_{\text {road_k }}\right) \in[0,1]$. The buffer is also evaluated upon each encounter with another node and if there is still a need to free the buffer, the oldest messages are discarded. Messages are deleted following one of the following policies. 1) Messaged lifetime as suggest by TTL expires 2) Node buffer becomes full 3) Probability of the vehicle is less than threshold probability.

\subsection{Simulation Environment}

We evaluate performance of road-aware geocast over a street map typical of roads in a metropolis. Roads consist of multi-lane directional highways with turns and intersections. We generate realistic mobility pattern of vehicles using Intelligent Driver Model (IDM) [21] car following and MOBIL (Minimizing Overall Braking Induced by Lane change) [22] lane change models. IDM is a traffic flow model and the decision of any vehicle driver to accelerate or to brake depends only on his or her own speed, and on the position and speed of the "leading vehicle" immediately ahead. However lane changing decisions depend on all neighboring vehicles. Various IDM parameters such as desired velocity, maximum acceleration and braking, minimum gap, and the minimum time headway, etc., can be customized through an XML configuration file. During each step, the Highway object passes information to the IDM model about the vehicle in front and the model determines what the current acceleration should be.

Highway related details such as highway id, number of lanes, direction, length, turn velocities etc., are specified using XML. Highway connections are created by defining associated front, back, left and right highways. Vehicle are generated (according to the desired density) by defining starting highway and multiple destination highways. Weight can be assigned to distribute percentages of generated vehicles on each destination road. Figure 8 shows the road network we used in the simulations.

We have implemented our road-aware geocast mechanism for VANETs using NS-3. Vehicles are configured to move with $40-120 \mathrm{~km} / \mathrm{h}$ speed towards randomly chosen destinations using shortest path which is calculated using dijkstra's algorithm. We developed a road-aware geocast application that creates and periodically broadcasts messages. Each message consists of message ID, TTL, timestamp, source vehicle ID, coordinates of the source vehicle, geographic region information (GR road IDs) and a message. We also implemented a geocast router application that implements broadcast and deletion policies.

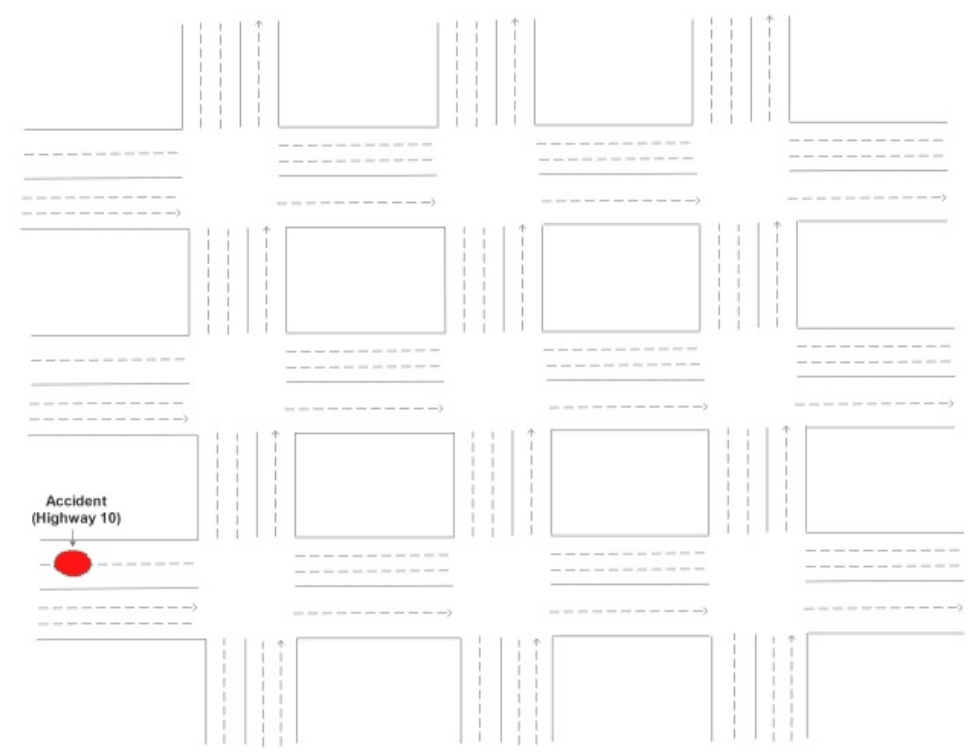

Figure 8. Road Network Used in Simulations

We create two vehicle mobility scenarios as shown in Table 1. We use OFDM 6Mbps data rate for wireless links (Wi-Fi ad hoc mode) and radio transmit power as $21 \mathrm{dBm}$ with Nakagami channel fading. Both transmit and receive antenna gains are fixed at $2 \mathrm{dBi}$. Energy detection threshold is set to $-101 \mathrm{dBm}$. Table 1 shows the simulation parameters used in our simulations. We use four vehicle density scenarios namely small (200 vehicles), medium (350 vehicles), large (500 vehicles) and very large (700 vehicles). 
Table 1. Simulation Scenarios

\begin{tabular}{|c|c|c|}
\hline \multicolumn{3}{|c|}{ Scenario 1} \\
\hline Speed (KPH) & \multicolumn{2}{|l|}{ Number of vehicles } \\
\hline \multirow{4}{*}{70} & & 200 \\
\hline & & 350 \\
\hline & & 500 \\
\hline & & 700 \\
\hline \multirow{4}{*}{100} & & 200 \\
\hline & & 350 \\
\hline & & 500 \\
\hline & & 700 \\
\hline \multicolumn{3}{|c|}{ Scenario 2} \\
\hline Number of vehicles & Speed (KPH) & \\
\hline \multirow{4}{*}{350} & & 40 \\
\hline & & 70 \\
\hline & & 100 \\
\hline & & 120 \\
\hline \multirow{4}{*}{500} & & 40 \\
\hline & & 70 \\
\hline & & 100 \\
\hline & & 120 \\
\hline
\end{tabular}

Table 2. Simulation parameters

\begin{tabular}{ll}
\hline Communication & NS 3 \\
\hline Simulator & OFDM \\
Modulation scheme & $21 \mathrm{dBm}$ \\
Transmission power & $6 \mathrm{Mbps}$ \\
Transmission rate & $-101 \mathrm{dBm}$ \\
Energy detection threshold & \\
\hline Vehicular Traffic & IDM \\
\hline Mobility Model & MOBIL \\
Lane Change Model & Highway \\
Road Scenario & Random \\
Number of lanes & 10 to 50 vehicle/km \\
Vehicular Density & 40 to $120 \mathrm{~km} / \mathrm{h}$ \\
Vehicle Speed &
\end{tabular}

We simulate a single source point. The accident vehicle generates and broadcasts warning message every second. All vehicles broadcast 1 hop hello messages every second. Hello messages signal a vehicle's presence and desire to get a copy of warning messages being shared in its road which can be geocast region of the warning message.

\subsection{Evaluation}

In the first series of simulations, we simulate our approach for two different speeds and 4 different vehicle densities. We evaluate feasibility and success of road-aware geocast for different road levels. Road levels are defined as the roads after the intersections. For example, 3rd road level means there are two intersections between the source vehicle road and the current road. We use road levels from the spanning tree and evaluate the successful message delivery ratio for each road level. Figure 9 and 10 show that our scheme successfully delivers the messages to vehicles on the roads using the probability based geocast region selection. In both the scenarios, delivery ratio is around $40 \%$ for the 4 th level of roads. As we use the threshold probability 0.05 , only first 4 road levels are selected as geocast regions. Roads after the 4th intersection are excluded from the geocast region due to the threshold. Delivery ratio increases with the increase in vehicle density. When the vehicle traffic is sparse, the delivery ratio is decreased because there are fewer vehicles to forward the messages. Similarly, the delivery ratio decreases with the increase in vehicle speeds. 


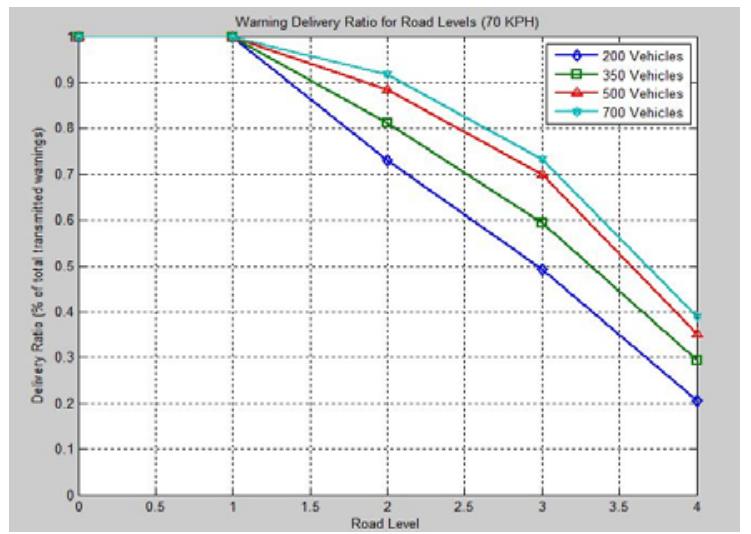

Figure 9. Delivery Ratio with 70kph Speed

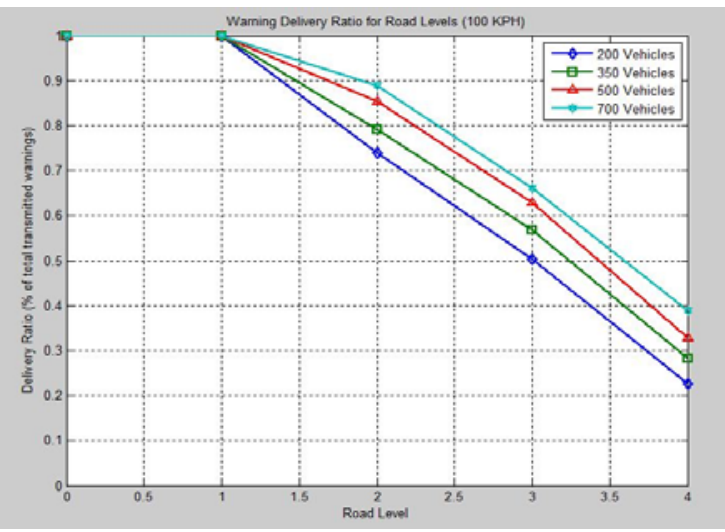

Figure 10. Delivery Ratio with 100kph Speed

In the second scenario we simulate our road-aware geocast mechanism for 4 different speeds of vehicles as in Table 1. Figure 11 and 12 show the results of our simulations and it can be clearly seen that increasing the speed while keeping the vehicle density constant, decrease the delivery ratio. In Figure 11 delivery ratio of different vehicle speeds with 350 vehicles is described. Our approach delivers around 30\% warning messages to the 4th level of roads from the spanning tree. Ratio of delivered messages is around $80 \%$ for the first level of roads which shows that our road-aware geocast mechanism exhibits high performance in highway VANET.

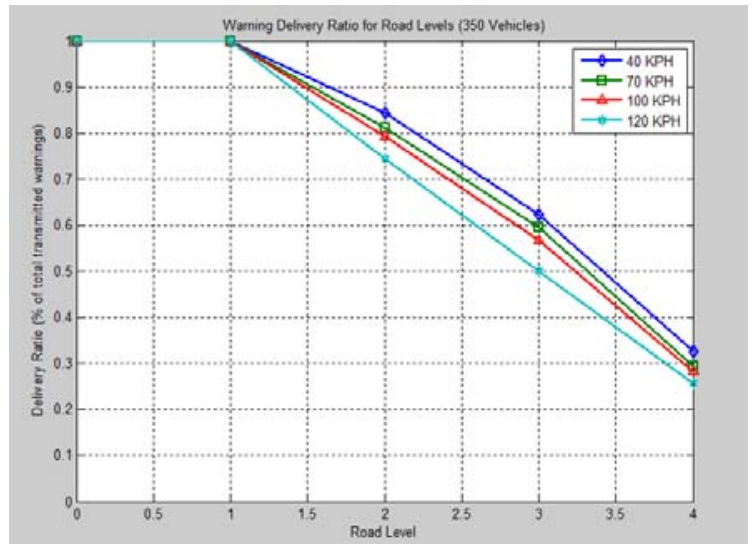

Figure 11. Delivery Ratio with 350 Vehicles 


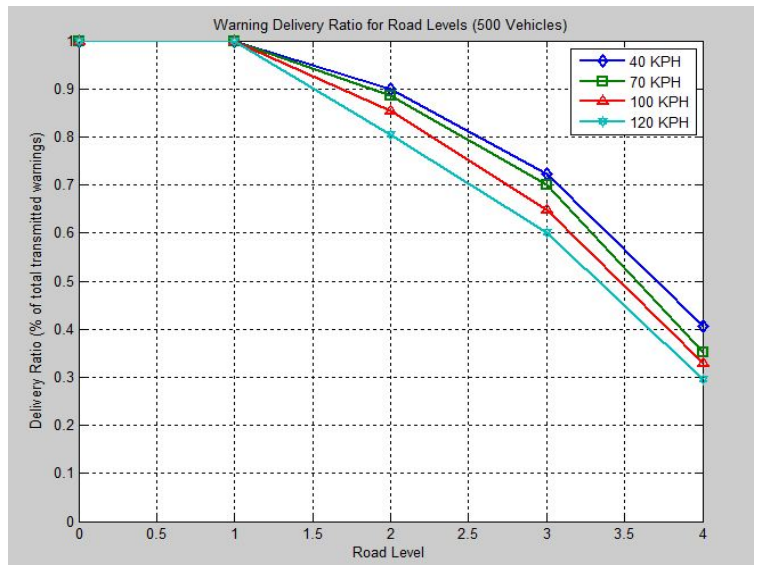

Figure 12. Delivery Ratio with 500 Vehicles

\section{CONCLUSIONS}

Vehicles caught up in the accident periodically broadcast warning messages that propagate in realtime to other vehicles in the neighbored. Information dissemination is geographically limited to vehicles in the neighborhood whose probability of going to the accident road so suggests. Location of accident vehicles serves as the source point and the locality where messages propagate is the geocast region for the messages. In our scheme, geocast region is not based on a circular region defined by a radius and a center point which is the case for geocast techniques used so far in MANETs/VANETs.

In order to define the geocast region, we construct a spanning tree of roads leading to the accident road. The tree is rooted at the accident vehicle(s) and intersections directly connected to the accident road are at level 1 of the tree. Level 1 intersections are then connected to further (i.e., level 2) intersections. Edges in the tree represent multi-lane two-way roads. Messages spread along the branches of the tree form the root towards the higher levels (or upstream roads with respect to the accident road).

We have experimented with various vehicle speeds and vehicle densities. According to the simulation results, our geocast mechanism is efficient and propagates information to only relevant vehicles within a VANET. Vehicles carrying communication devices with moderate compute/storage resources and network bandwidth can benefit from the solution. Our solution can help travelers reduce their travel times and cut fuel costs by helping them avoid traffic jams, accidents and obstacles.

\section{ACKNOWLEDGEMENTS}

This research was supported by Basic Science Research Program through the National Research Foundationof Korea (NRF) funded by the Ministry of Education, Science, and Technology (2011-0012329).

\section{REFERENCES}

[1] T. Little and A. Agarwal. "An information propagation scheme for VANETS”, MCL Technical Report 07-01-2005, Department of Elecrtical and Computer Engineering, Boston University, USA, July 2005.

[2] Q. Sun and G. Hector. "Using Ad-Hoc Inter-vechicle Networks for Regional Alerts”, TR, Stanford University, October 2004.

[3] A. Xeros, M. Andreou, A. Pitsillides and M. Lestas. "Information Propagation Probability on Intersections in VANETs”, In Third Inter. Workshop on Vehicle-to-Vehicle Communications, June, 2007.

[4] A.Xeros, M. Andreou, M. Lestas, G. Papageorgiou, V. Vassiliou and A.Pitsillides. "Time Constrained Message Delivery Probability in VANETS”, in Proc., of the 4th Vehicle to Vehicle Communication workshop, V2Vcom 2008.

[5] N. Dragos and B. Nath. "Trajectory based Forwarding and its Applications", In the Proc. of the ACM International Conference on Mobile Computing and Networking (MOBICOM’03), USA, 2003.

[6] C. Lochert, M. Mauve, H. Fubler and H. Hartenstein. "Geographic Routing in City Scenarios”, In the Proc. of the ACM SIGMOBILE Mobile Computing and Communications Review (MC2R) 9(1), 2005.

[7] C. Lochert, M. Mauve, H. Fubler and H. Hartenstein. "On Utility-Fair Broadcast in VANETS”, In the Proc. of the 2nd Intern. Workshop on Intelligent Transportation (WIT 2005) Germany, 2005.

[8] L. Wischhof, A. Ebner and H. Rohling. "Information dissemination in Self-Organizing Intervehicle Networks", IEEE Transactions on Intelligent Transportation Systems, 6(1), pp. 90-101, March 2005. 
[9] Maihofer, Christian. "A survey of geocast routing protocols." Communications Surveys \& Tutorials, IEEE 6.2 (2004): 32-42.

[10] Ko, Young Bae, and Nitin H. Vaidya. "Flooding-based geocasting protocols for mobile ad hoc networks". Mobile Networks and Applications 7.6 (2002): 471-480.

[11] Celes, C., Braga, R. B., De Oliveira, C. T., Andrade, R., \&Loureiro, A. A. (2013, September). GeoSPIN: An Approach for Geocast Routing Based on SPatialINformation in VANETs. In Vehicular Technology Conference (VTC Fall), 2013 IEEE 78th (pp. 1-6). IEEE.

[12] Akamatsu, R., Suzuki, M., Okamoto, T., Hara, K., \&Shigeno, H. (2014, January). Adaptive delay-based geocast protocol for data dissemination in urban VANET. In Mobile Computing and Ubiquitous Networking (ICMU), 2014 Seventh International Conference on (pp. 141-146). IEEE.

[13] Zhang, Lei, Boyang Yu, and Jianping Pan. "GeoMob: A mobility-aware geocast scheme in metropolitans via taxicabs and buses”. INFOCOM, 2014 Proceedings IEEE. IEEE, 2014.

[14] Rahbar, Hamidreza, KshirasagarNaik, and AmiyaNayak. "DTSG: Dynamic time-stable geocast routing in vehicular ad hoc networks”. Ad Hoc Networking Workshop (Med-Hoc-Net), 2010 The 9th IFIP Annual Mediterranean. IEEE, 2010.

[15] Henderson, Thomas R., Mathieu Lacage, George F. Riley, C. Dowell, and J. B. Kopena. "Network simulations with the ns-3 simulator”. SIGCOMM demonstration (2008).

[16] Li, Fan, and Yu Wang. "Routing in vehicular ad hoc networks: A survey". Vehicular Technology Magazine, IEEE 2.2 (2007): 12-22.

[17] Henderson, Thomas R., Mathieu Lacage, George F. Riley, C. Dowell, and J. B. Kopena. "Network simulations with the ns-3 simulator”. SIGCOMM demonstration (2008).

[18] Allal, Salim, and Saadi Boudjit. "Geocast routing protocols for vanets: Survey and guidelines”. Innovative Mobile and Internet Services in Ubiquitous Computing (IMIS), 2012 Sixth International Conference on. IEEE, 2012.

[19] Jochle, Timm, BjörnWiedersheim, Florian Schaub, and Michael Weber. "Efficiency analysis of geocast target region specifications for VANET applications”. In VNC, pp. 250-257. 2012.

[20] Rahbar, Hamidreza. "Dynamic time-stable geocast routing in vehicular ad hoc networks”. (2010)

[21] Treiber, Martin, Ansgar Hennecke, and Dirk Helbing. "Congested traffic states in empirical observations and microscopic simulations”. Physical Review E62.2 (2000): 1805.

[22] Treiber, Martin, and Arne Kesting. "Modeling lane-changing decisions with MOBIL". Traffic and Granular Flow’07. Springer Berlin Heidelberg, 2009. 211-221.

\section{BIOGRAPHIES OF AUTHORS}

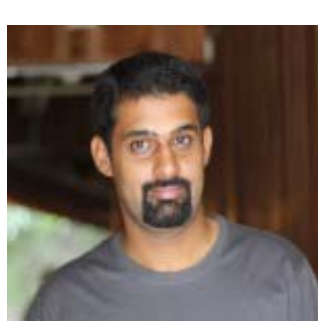

Zubair Amjad received the B.S. degree in telecommunication engineering from National University of Computer and Emerging Sciences, Islamabad, Pakistan, in 2012. He is a research student in department of computer engineering, Jeju Nation University, South Korea. His interests are in vehicular ad hoc networks and software defines networks.

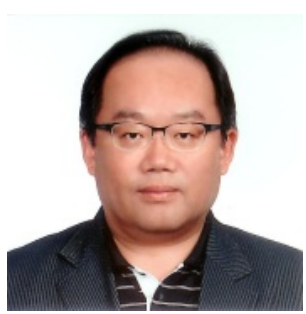

Wang-Cheol Song received the B.S. degree in Food Engineering and Electronics from Yonsei University, Seoul, Korea in 1986 and 1989, respectively. And M.S. and PhD in Electronics studies from Yonsei University, Seoul, Korea, in 1991 and 1995, respectively. From 1996 he has worked for Jeju National University. His research interests include VANETs and MANETs, Software Defined Networks, network security, and network management. 